\title{
Incremental generation of spatial referring expressions in situated dialog*
}

\author{
John D. Kelleher \\ Dublin Institute of Technology \\ Dublin, Ireland \\ john.kelleherdcomp.dit.ie
}

\author{
Geert-Jan M. Kruijff \\ DFKI GmbH \\ Saarbrücken, Germany \\ gjedfki.de
}

\begin{abstract}
This paper presents an approach to incrementally generating locative expressions. It addresses the issue of combinatorial explosion inherent in the construction of relational context models by: (a) contextually defining the set of objects in the context that may function as a landmark, and (b) sequencing the order in which spatial relations are considered using a cognitively motivated hierarchy of relations, and visual and discourse salience.
\end{abstract}

\section{Introduction}

Our long-term goal is to develop conversational robots with whom we can interact through natural, fluent, visually situated dialog. An inherent aspect of visually situated dialog is reference to objects located in the physical environment (Moratz and Tenbrink, 2006). In this paper, we present a computational approach to the generation of spatial locative expressions in such situated contexts.

The simplest form of locative expression is a prepositional phrase, modifying a noun phrase to locate an object. (1) illustrates the type of locative we focus on generating. In this paper we use the term target $(\mathrm{T})$ to refer to the object that is being located by a spatial expression and the term landmark (L) to refer to the object relative to which the target's location is described.

\section{(1) a. the book $[\mathrm{T}]$ on the table $[\mathrm{L}]$}

Generating locative expressions is part of the general field of generating referring expressions (GRE). Most GRE algorithms deal with the same problem: given a domain description and a target object, generate a description of the target object that distinguishes it from the other objects in the domain. We use distractor objects to indicate the

The research reported here was supported by the CoSy project, EU FP6 IST "Cognitive Systems" FP6-004250-IP. objects in the context excluding the target that at a given point in processing fulfill the description of the target object that has been generated. The description generated is said to be distinguishing if the set of distractor objects is empty.

Several GRE algorithms have addressed the issue of generating locative expressions (Dale and Haddock, 1991; Horacek, 1997; Gardent, 2002; Krahmer and Theune, 2002; Varges, 2004). However, all these algorithms assume the GRE component has access to a predefined scene model. For a conversational robot operating in dynamic environments this assumption is unrealistic. If a robot wishes to generate a contextually appropriate reference it cannot assume the availability of a fixed scene model, rather it must dynamically construct one. However, constructing a model containing all the relationships between all the entities in the domain is prone to combinatorial explosion, both in terms of the number objects in the context (the location of each object in the scene must be checked against all the other objects in the scene) and number of inter-object spatial relations (as a greater number of spatial relations will require a greater number of comparisons between each pair of objects). ${ }^{1}$ Also, the context free a priori construction of such an exhaustive scene model is cognitively implausible. Psychological research indicates that spatial relations are not preattentively perceptually available (Treisman and Gormican, 1988), their perception requires attention (Logan, 1994; Logan, 1995). Subjects appear to construct contextually dependent reduced relational scene models, not exhaustive context free models.

Contributions We present an approach to in-

\footnotetext{
${ }^{1}$ In English, the vast majority of spatial locatives are binary, some notable exceptions include: between, amongst etc. However, we will not deal with these exceptions in this paper.
} 
crementally generating locative expressions. It addresses the issue of combinatorial explosion inherent in relational scene model construction by incrementally creating a series of reduced scene models. Within each scene model only one spatial relation is considered and only a subset of objects are considered as candidate landmarks. This reduces both the number of relations that must be computed over each object pair and the number of object pairs. The decision as to which relations should be included in each scene model is guided by a cognitively motivated hierarchy of spatial relations. The set of candidate landmarks in a given scene is dependent on the set of objects in the scene that fulfil the description of the target object and the relation that is being considered.

Overview $\S 2$ presents some relevant background data. $\S 3$ presents our GRE approach. $\S 4$ illustrates the framework on a worked example and expands on some of the issues relevant to the framework. We end with conclusions.

\section{Data}

If we consider that English has more than eighty spatial prepositions (omitting compounds such as right next to) (Landau, 1996), the combinatorial aspect of relational scene model construction becomes apparent. It should be noted that for our purposes, the situation is somewhat easier because a distinction can be made between static and dynamic prepositions: static prepositions primarily ${ }^{2}$ denote the location of an object, dynamic prepositions primarily denote the path of an object (Jackendoff, 1983; Herskovits, 1986), see (2). However, even focusing just on the set of static prepositions does not remove the combinatorial issues effecting the construction of a scene model.

(2) a. the tree is behind [static] the house

b. the man walked across [dyn.] the road

In general, static prepositions can be divided into two sets: topological and projective. Topological prepositions are the category of prepositions referring to a region that is proximal to the landmark; e.g., at, near, etc. Often, the distinctions between the semantics of the different topological prepositions is based on pragmatic contraints, e.g. the use of at licences the target to be

\footnotetext{
${ }^{2}$ Static prepositions can be used in dynamic contexts, e.g. the man ran behind the house, and dynamic prepositions can be used in static ones, e.g. the tree lay across the road.
}

in contact with the landmark, whereas the use of near does not. Projective prepositions describe a region projected from the landmark in a particular direction; e.g., to the right of, to the left of. The specification of the direction is dependent on the frame of reference being used (Herskovits, 1986).

Static prepositions have both qualitative and quantitative semantic properties. The qualitative aspect is evident when they are used to denote an object by contrasting its location with that of the distractor objects. Using Figure 1 as visual context, the locative expression the circle on the left of the square illustrates the contrastive semantics of a projective preposition, as only one of the circles in the scene is located in that region. Taking Figure 2, the locative expression the circle near the black square shows the contrastive semantics of a topological preposition. Again, of the two circles in the scene only one of them may be appropriately described as being near the black square, the other circle is more appropriately described as being near the white square. The quantitative aspect is evident when a static preposition denotes an object using a relative scale. In Figure 3 the locative the circle to the right of the square shows the relative semantics of a projective preposition. Although both the circles are located to the right of the square we can distinguish them based on their location in the region. Figure 3 also illustrates the relative semantics of a topological preposition Figure 3. We can apply a description like the circle near the square to either circle if none other were present. However, if both are present we can interpret the reference based on relative proximity to the landmark the square.

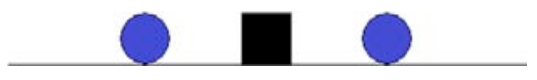

Figure 1: Visual context illustrating contrastive semantics of projective prepositions

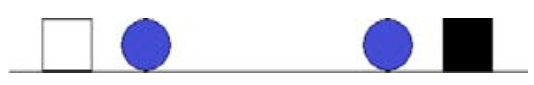

Figure 2: Visual context illustrating contrastive semantics of topological prepositions

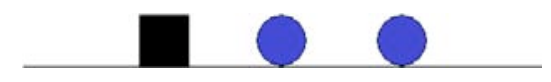

Figure 3: Visual context illustrating relative semantics of topological and projective prepositions 


\section{Approach}

We base our GRE approach on an extension of the incremental algorithm (Dale and Reiter, 1995). The motivation for basing our approach on this algorithm is its polynomial complexity. The algorithm iterates through the properties of the target and for each property computes the set of distractor objects for which (a) the conjunction of the properties selected so far, and (b) the current property hold. A property is added to the list of selected properties if it reduces the size of the distractor object set. The algorithm succeeds when all the distractors have been ruled out, it fails if all the properties have been processed and there are still some distractor objects. The algorithm can be refined by ordering the checking of properties according to fixed preferences, e.g. first a taxonomic description of the target, second an absolute property such as colour, third a relative property such as size. (Dale and Reiter, 1995) also stipulate that the type description of the target should be included in the description even if its inclusion does not make the target distinguishable.

We extend the original incremental algorithm in two ways. First we integrate a model of object salience by modifying the condition under which a description is deemed to be distinguishing: it is, if all the distractors have been ruled out or if the salience of the target object is greater than the highest salience score ascribed to any of the current distractors. This is motivated by the observation that people can easily resolve underdetermined references using salience (Duwe and Strohner, 1997). We model the influence of visual and discourse salience using a function salience $(L)$, Equation 1. The function returns a value between 0 and 1 to represent the relative salience of a landmark $L$ in the scene. The relative salience of an object is the average of its visual salience $\left(S_{v i s}\right)$ and discourse salience $\left(S_{\text {disc }}\right)$,

$$
\text { salience }(L)=\left(S_{\text {vis }}(L)+S_{d i s c}(L)\right) / 2
$$

Visual salience $S_{\text {vis }}$ is computed using the algorithm of (Kelleher and van Genabith, 2004). Computing a relative salience for each object in a scene is based on its perceivable size and its centrality relative to the viewer focus of attention, returning scores in the range of 0 to 1 . The discourse salience $\left(S_{d i s c}\right)$ of an object is computed based on recency of mention (Hajicová, 1993) except we represent the maximum overall salience in the scene as 1 , and use 0 to indicate that the landmark is not salient in the current context. Algorithm 1 gives the basic algorithm with salience.

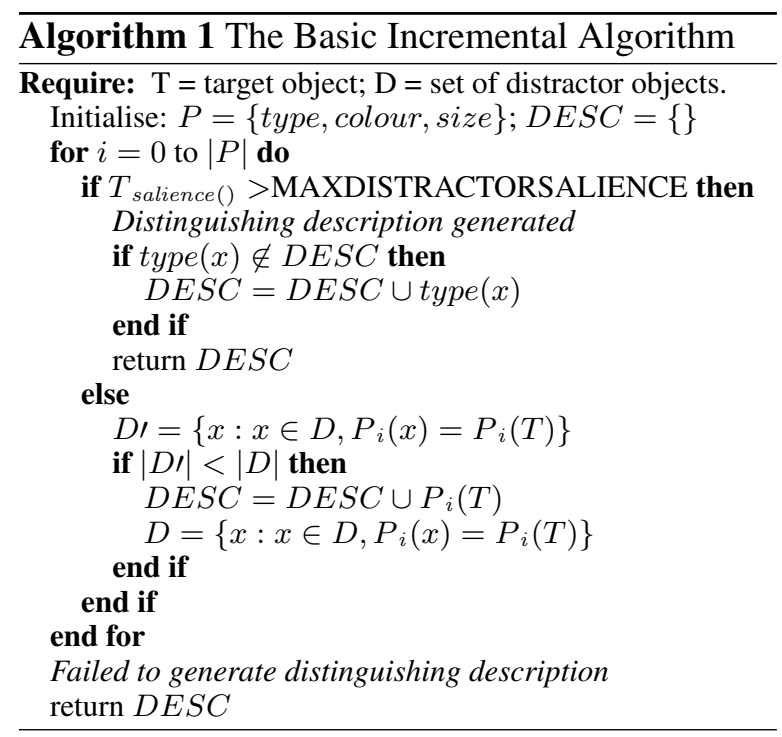

Secondly, we extend the incremental algorithm in how we construct the context model used by the algorithm. The context model determines to a large degree the output of the incremental algorithm. However, Dale and Reiter do not define how this set should be constructed, they only write: "[w]e define the context set to be the set of entities that the hearer is currently assumed to be attending to" (Dale and Reiter, 1995, pg. 236).

Before applying the incremental algorithm we must construct a context model in which we can check whether or not the description generated distinguishes the target object. To constrain the combinatorial explosion in relational scene model construction we construct a series of reduced scene models, rather than one complex exhaustive model. This construction is driven by a hierarchy of spatial relations and the partitioning of the context model into objects that may and may not function as landmarks. These two components are developed below. $\S 3.1$ discusses a hierarchy of spatial relations, and $\S 3.2$ presents a classification of landmarks and uses these groupings to create a definition of a distinguishing locative description. In $\S 3.3$ we give the generation algorithm integrating these components.

\subsection{Cognitive Ordering of Contexts}

Psychological research indicates that spatial relations are not preattentively perceptually available (Treisman and Gormican, 1988). Rather, their perception requires attention (Logan, 1994; 
Logan, 1995). These findings point to subjects constructing contextually dependent reduced relational scene models, rather than an exhaustive context free model. Mimicking this, we have developed an approach to context model construction that constrains the combinatorial explosion inherent in the construction of relational context models by incrementally building a series of reduced context models. Each context model focuses on a different spatial relation. The ordering of the spatial relations is based on the cognitive load of interpreting the relation. Below we motivate and develop the ordering of relations used.

We can reasonably asssume that it takes less effort to describe one object than two. Following the Principle of Minimal Cooperative Effort (Clark and Wilkes-Gibbs, 1986), one should only use a locative expression when there is no distinguishing description of the target object using a simple feature based approach. Also, the Principle of Sensitivity (Dale and Reiter, 1995) states that when producing a referring expression, one should prefer features the hearer is known to be able to interpret and see. This points to a preference, due to cognitive load, for descriptions that identify an object using purely physical and easily perceivable features ahead of descriptions that use spatial expressions. Experimental results support this (van der Sluis and Krahmer, 2004).

Similarly, we can distinguish between the cognitive loads of processing different forms of spatial relations. In comparing the cognitive load associated with different spatial relations it is important to recognize that they are represented and processed at several levels of abstraction. For example, the geometric level, where metric properties are dealt with, the functional level, where the specific properties of spatial entities deriving from their functions in space are considered, and the pragmatic level, which gathers the underlying principles that people use in order to discard wrong relations or to deduce more information (Edwards and Moulin, 1998). Our discussion is grounded at the geometric level.

Focusing on static prepositions, we assume topological prepositions have a lower perceptual load than projective ones, as perceiving two objects being close to each other is easier than the processing required to handle frame of reference ambiguity (Carlson-Radvansky and Irwin, 1994; Carlson-Radvansky and Logan,
1997). Figure 4 lists the preferences, further discerning objects type as the easiest to process, before absolute gradable predicates (e.g. color), which is still easier than relative gradable predicates

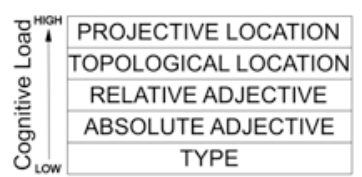

Figure 4: Cognitive load (e.g. size) (Dale and Reiter, 1995).

We can refine the topological versus projective preference further if we consider their contrastive and relative uses of these relations ( $\$ 2$ ). Perceiving and interpreting a contrastive use of a spatial relation is computationally easier than judging a relative use. Finally, within projective prepositions, psycholinguistic data indicates a perceptually based ordering of the relations: abovelbelow are easier to percieve and interpret than in front of /behind which in turn are easier than to the right of/to the left of (Bryant et al., 1992; Gapp, 1995). In sum, we propose the following ordering: topological contrastive $<$ topological relative $<$ projective constrastive $<$ projective relative.

For each level of this hierarchy we require a computational model of the semantics of the relation at that level that accomodates both contrastive and relative representations. In $\S 2$ we noted that the distinctions between the semantics of the different topological prepositions is often based on functional and pragmatic issues. ${ }^{3}$ Currently, however, more psycholinguistic data is required to distinguish the cognitive load associated with the different topological prepositions. We use the model of topological proximity developed in (Kelleher et al., 2006) to model all the relations at this level. Using this model we can define the extent of a region proximal to an object. If the target or one of the distractor objects is the only object within the region of proximity around a given landmark this is taken to model a contrastive use of a topological relation relative to that landmark. If the landmark's region of proximity contains more than one object from the target and distractor object set then it is a relative use of a topological relation. We handle the issue of frame of reference ambiguity and model the semantics of projective prepostions using the framework developed in (Kelleher et al., 2006). Here again, the contrastive-relative distinc-

\footnotetext{
${ }^{3}$ See inter alia (Talmy, 1983; Herskovits, 1986; Vandeloise, 1991; Fillmore, 1997; Garrod et al., 1999) for more discussion on these differences
} 
tion is dependent on the number of objects within the region of space defined by the preposition.

\subsection{Landmarks and Descriptions}

If we want to use a locative expression, we must choose another object in the scene to function as landmark. An implicit assumption in selecting a landmark is that the hearer can easily identify and locate the object within the context. A landmark can be: the speaker (3)a, the hearer (3)b, the scene (3)c, an object in the scene (3)d, or a group of objects in the scene (3)e. ${ }^{4}$

(3) a. the ball on my right [speaker]

b. the ball to your left [hearer]

c. the ball on the right [scene]

d. the ball to the left of the box [an object in the scene]

e. the ball in the middle [group of objects]

Currently, we need new empirical research to see if there is a preference order between these landmark categories. Intuitively, in most situations, either of the interlocutors are ideal landmarks because the speaker can naturally assume that the hearer is aware of the speaker's location and their own. Focusing on instances where an object in the scene is used as a landmark, several authors (Talmy, 1983; Landau, 1996; Gapp, 1995) have noted a target-landmark asymmetry: generally, the landmark object is more permanently located, larger, and taken to have greater geometric complexity. These characteristics are indicative of salient objects and empirical results support this correlation between object salience and landmark selection (Beun and Cremers, 1998). However, the salience of an object is intrinsically linked to the context it is embedded in. For example, in Figure 5 the ball has a relatively high salience, because it is a singleton, despite the fact that it is smaller and geometrically less complex than the other figures. Moreover, in this scene it is the only object that can function as a landmark without recourse to using the scene itself or a grouping of objects.

Clearly, deciding which objects in a given context are suitable to function as landmarks is a complex and contextually dependent process. Some of the factors effecting this decision are object

\footnotetext{
${ }^{4}$ See (Gorniak and Roy, 2004) for further discussion on the use of spatial extrema of the scene and groups of objects in the scene as landmarks
}

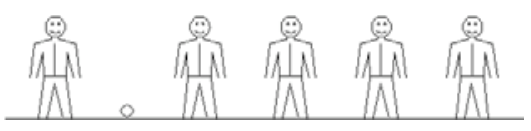

Figure 5: Landmark salience

salience and the functional relationships between objects. However, one basic constraint on landmark selection is that the landmark should be distinguishable from the target. For example, given the context in Figure 5 and all other factors being equal, using a locative such as the man to the left of the man would be much less helpful than using the man to the right of the ball. Following this observation, we treat an object as a candidate landmark if the following conditions are met: (1) the object is not the target, and (2) it is not in the distractor set either.

Furthermore, a target landmark is a member of the candidate landmark set that stands in relation to the target. A distractor landmark is a member of the candidate landmark set that stands in the considered relation to a distractor object. We then define a distinguishing locative description as a locative description where there is target landmark that can be distinguished from all the members of the set of distractor landmarks under the relation used in the locative.

\subsection{Algorithm}

We first try to generate a distinguishing description using Algorithm 1. If this fails, we divide the context into three components: the target, the distractor objects, and the set of candidate landmarks. We then iterate through the set of candidate landmarks (using a salience ordering if there is more than one, cf. Equation 1) and try to create a distinguishing locative description. The salience ordering of the landmarks is inspired by (Conklin and McDonald, 1982) who found that the higher the salience of an object the more likely it appears in the description of the scene it was embedded in. For each candidate landmark we iterate through the hierarchy of relations, checking for each relation whether the candidate can function as a target landmark under that relation. If so we create a context model that defines the set of target and distractor landmarks. We create a distinguishing locative description by using the basic incremental algorithm to distinguish the target landmark from the distractor landmarks. If we succeed in generating a distinguishing locative description we return 
the description and stop.

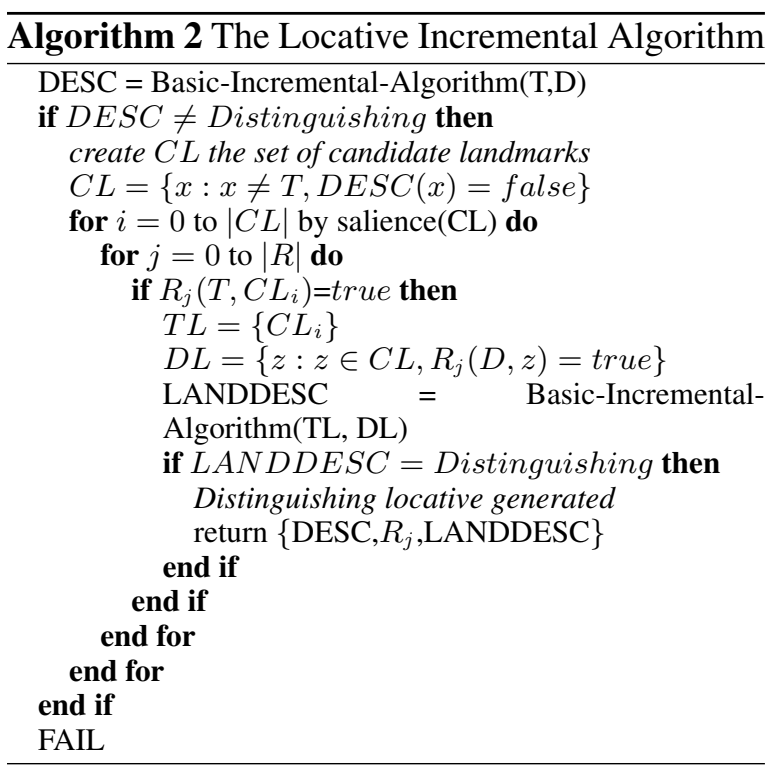

If we cannot create a distinguishing locative description we face two choices: (1) iterate on to the next relation in the hierarchy, (2) create an embedded locative description distinguishing the landmark. We adopt (1) over (2), preferring the dog to the right of the car over the dog near the car to the right of the house. However, we can generate these longer embedded descriptions if needed, by replacing the call to the basic incremental algorithm for the landmark object with a call to the whole locative expression generation algorithm, using the target landmark as the target object and the set of distractor landmarks as the distractors.

An important point in this context is the issue of infinite regression (Dale and Haddock, 1991). A compositional GRE system may in certain contexts generate an infinite description, trying to distinguish the landmark in terms of the target, and the target in terms of the landmark, cf. (4). But, this infinite recursion can only occur if the context is not modified between calls to the algorithm. This issue does not affect Algorithm 2 as each call to the algorithm results in the domain being partitioned into those objects we can and cannot use as landmarks. This not only reduces the number of object pairs that relations must be computed for, but also means that we need to create a distinguishing description for a landmark on a context that is a strict subset of the context the target description was generated in. This way the algorithm cannot distinguish a landmark using its target.

(4) the bowl on the table supporting the bowl on the table supporting the bowl ...

\subsection{Complexity}

The computational complexity of the incremental algorithm is $\mathrm{O}\left(n_{d} * n_{l}\right)$, with $n_{d}$ the number of distractors, and $n_{l}$ the number of attributes in the final referring description (Dale and Reiter, 1995). This complexity is independent of the number of attributes to be considered. Algorithm 2 is bound by the same complexity. For the average case, however, we see the following. For one, with every increase in $n_{l}$, we see a strict decrease in $n_{d}$ : the more attributes we need, the fewer distractors we strictly have due to the partitioning into distractor and target landmarks. On the other hand, we have the dynamic construction of a context model. This latter factor is not considered in (Dale and Reiter, 1995), meaning we would have to multiply $\mathrm{O}\left(n_{d} * n_{l}\right)$ with a constant $K_{c t x t}$ for context construction. Depending on the size of this constant, we may see an advantage of our algorithm in that we only consider a single spatial relation each time we construct a context model, we avoid an exponential number of comparisons: we need to make at most $n_{d} *\left(n_{d}-1\right)$ comparisons (and only $n_{d}$ if relations are symmetric).

\section{Discussion}

We examplify the approach on the visual scene on the left of Figure 6. This context consists of two red boxes R1 and R2 and two blue balls B1 and B2. Imagine that we want to refer to B1. We begin by calling Algorithm 2. This in turn calls Algorithm 1, returning the property ball. This is not sufficient to create a distinguishing description as B2 is also a ball. In this context the set of candidate landmarks equals $\{\mathrm{R} 1, \mathrm{R} 2\}$. We take $\mathrm{R} 1$ as first candidate landmark, and check for topological proximity in the scene as modeled in (Kelleher et al., 2006). The image on the right of Figure 6 illustrates the resulting scene analysis: the green region on the left defines the area deemed to be proximal to $\mathrm{R} 1$, and the yellow region on the right defines the area proximal to R2. Clearly, B1 is in the area proximal to $\mathrm{R} 1$, making $\mathrm{R} 1$ a target landmark. As none of the distractors (i.e., B2) are located in a region that is proximal to a candidate landmark there are no distractor landmarks. As a result when the basic incremental algorithm is called to create a distinguishing description for the target landmark R1 it will return box and this will be deemed to be a distinguishing locative description. The overall algorithm will then return 


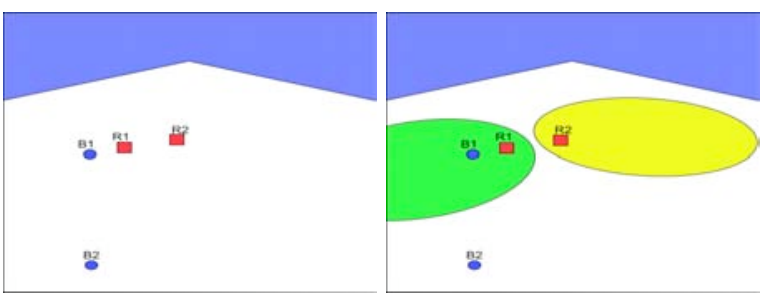

Figure 6: A visual scene and the topological analsis of R1 and R2

the vector $\{$ ball, proximal, box $\}$ which would result in the realiser generating a reference of the form: the ball near the box. ${ }^{5}$

The relational hierarchy used by the framework has some commonalities with the relational subsumption hierarchy proposed in (Krahmer and Theune, 2002). However, there are two important differences between them. First, an implication of the subsumption hierarchy proposed in (Krahmer and Theune, 2002) is that the semantics of the relations at lower levels in the hierarchy are subsumed by the semantics of their parent relations. For example, in the portion of the subsumption hierarchy illustrated in (Krahmer and Theune, 2002) the relation next to subsumes the relations left of and right of. By contrast, the relational hierarchy developed here is based solely on the relative cognitive load associated with the semantics of the spatial relations and makes not claims as to the semantic relationships between the semantics of the spatial relations. Secondly, (Krahmer and Theune, 2002) do not use their relational hierarchy to guide the construction of domain models.

By providing a basic contextual definition of a landmark we are able to partition the context in an appropriate manner. This partitioning has two advantages. One, it reduces the complexity of the context model construction, as the relationships between the target and the distractor objects or between the distractor objects themselves do not need to be computed. Two, the context used during the generation of a landmark description is always a subset of the context used for a target (as the target, its distractors and the other objects in the domain that do not stand in relation to the target or distractors under the relation being considered are excluded). As a result the framework avoids the issue of infinite recusion. Furthermore, the target-landmark relationship is automat-

\footnotetext{
${ }^{5}$ For more examples, see the videos available at http://www.dfki.de/cosy/media/.
}

ically included as a property of the landmark as its feature based description need only distinguish it from objects that stand in relation to one of the distractor objects under the same spatial relationship.

In future work we will focus on extending the framework to handle some of the issues effecting the incremental algorithm, see (van Deemter, 2001). For example, generating locative descriptions containing negated relations, conjunctions of relations and involving sets of objects (sets of targets and landmarks).

\section{Conclusions}

We have argued that an if a conversational robot functioning in dynamic partially known environments needs to generate contextually appropriate locative expressions it must be able to construct a context model that explicitly marks the spatial relations between objects in the scene. However, the construction of such a model is prone to the issue of combinatorial explosion both in terms of the number objects in the context (the location of each object in the scene must be checked against all the other objects in the scene) and number of inter-object spatial relations (as a greater number of spatial relations will require a greater number of comparisons between each pair of objects.

We have presented a framework that addresses this issue by: (a) contextually defining the set of objects in the context that may function as a landmark, and (b) sequencing the order in which spatial relations are considered using a cognitively motivated hierarchy of relations. Defining the set of objects in the scene that may function as a landmark reduces the number of object pairs that a spatial relation must be computed over. Sequencing the consideration of spatial relations means that in each context model only one relation needs to be checked and in some instances the agent need not compute some of the spatial relations, as it may have succeeded in generating a distinguishing locative using a relation earlier in the sequence.

A further advantage of our approach stems from the partitioning of the context into those objects that may function as a landmark and those that may not. As a result of this partitioning the algorithm avoids the issue of infinite recursion, as the partitioning of the context stops the algorithm from distinguishing a landmark using its target.

We have employed the approach in a system for Human-Robot Interaction, in the setting of object 
manipulation in natural scenes. For more detail, see (Kruijff et al., 2006a; Kruijff et al., 2006b).

\section{References}

R.J. Beun and A. Cremers. 1998. Object reference in a shared domain of conversation. Pragmatics and Cognition, 6(1/2):121-152.

D.J. Bryant, B. Tversky, and N. Franklin. 1992. Internal and external spatial frameworks representing described scenes. Journal of Memory and Language, 31:74-98.

L.A. Carlson-Radvansky and D. Irwin. 1994. Reference frame activation during spatial term assignment. Journal of Memory and Language, 33:646-671.

L.A. Carlson-Radvansky and G.D. Logan. 1997. The influence of reference frame selection on spatial template construction. Journal of Memory and Language, 37:411-437.

H. Clark and D. Wilkes-Gibbs. 1986. Referring as a collaborative process. Cognition, 22:1-39.

E. Jeffrey Conklin and David D. McDonald. 1982. Salience: the key to the selection problem in natural language generation. In ACL Proceedings, 20th Annual Meeting, pages 129-135.

R. Dale and N. Haddock. 1991. Generating referring expressions involving relations. In Proceeding of the Fifth Conference of the European ACL, pages 161-166, Berlin, April.

R. Dale and E. Reiter. 1995. Computational interpretations of the Gricean maxims in the generation of referring expressions. Cognitive Science, 19(2):233-263.

I. Duwe and H. Strohner. 1997. Towards a cognitive model of linguistic reference. Report: 97/1 - Situierte Künstliche Kommunikatoren 97/1, Univeristät Bielefeld.

G. Edwards and B. Moulin. 1998. Towards the simulation of spatial mental images using the voronoï model. In P. Oliver and K.P. Gapp, editors, Representation and processing of spatial expressions, pages 163-184. Lawrence Erlbaum Associates.

C. Fillmore. 1997. Lecture on Deixis. CSLI Publications.

K.P. Gapp. 1995. Angle, distance, shape, and their relationship to projective relations. In Proceedings of the 17th Conference of the Cognitive Science Society.

C Gardent. 2002. Generating minimal definite descriptions. In Proceedings of the 40th International Confernce of the Association of Computational Linguistics (ACL-02), pages 96-103.

S. Garrod, G. Ferrier, and S. Campbell. 1999. In and on: investigating the functional geometry of spatial prepositions. Cognition, 72:167-189.

P. Gorniak and D. Roy. 2004. Grounded semantic composition for visual scenes. Journal of Artificial Intelligence Research, 21:429-470.

E. Hajicová. 1993. Issues of sentence structure and discourse patterns. In Theoretical and Computational Linguistics, volume 2, Charles University, Prague.

A Herskovits. 1986. Language and spatial cognition: An interdisciplinary study of prepositions in English. Studies in Natural Language Processing. Cambridge University Press.
H. Horacek. 1997. An algorithm for generating referential descriptions with flexible interfaces. In Proceedings of the 35th Annual Meeting of the Association for Computational Linguistics, Madrid.

R. Jackendoff. 1983. Semantics and Cognition. Current Studies in Linguistics. The MIT Press.

J. Kelleher and J. van Genabith. 2004. A false colouring real time visual salency algorithm for reference resolution in simulated 3d environments. AI Review, 21(3-4):253-267.

J.D. Kelleher, G.J.M. Kruijff, and F. Costello. 2006. Proximity in context: An empirically grounded computational model of proximity for processing topological spatial expressions. In Proceedings ACL/COLING 2006.

E. Krahmer and M. Theune. 2002. Efficient context-sensitive generation of referring expressions. In K. van Deemter and R. Kibble, editors, Information Sharing: Reference and Presupposition in Language Generation and Interpretation. CLSI Publications, Standford.

G.J.M. Kruijff, J.D. Kelleher, G. Berginc, and A. Leonardis. 2006a. Structural descriptions in human-assisted robot visual learning. In Proceedings of the 1st Annual Conference on Human-Robot Interaction (HRI'O6).

G.J.M. Kruijff, J.D. Kelleher, and Nick Hawes. 2006b. Information fusion for visual reference resolution in dynamic situated dialogue. In E. André, L. Dybkjaer, W. Minker, H.Neumann, and M. Weber, editors, Perception and Interactive Technologies (PIT 2006). Springer Verlag.

B Landau. 1996. Multiple geometric representations of objects in language and language learners. In P Bloom, M. Peterson, L Nadel, and M. Garrett, editors, Language and Space, pages 317-363. MIT Press, Cambridge.

G. D. Logan. 1994. Spatial attention and the apprehension of spatial realtions. Journal of Experimental Psychology: Human Perception and Performance, 20:1015-1036.

G.D. Logan. 1995. Linguistic and conceptual control of visual spatial attention. Cognitive Psychology, 12:523-533.

R. Moratz and T. Tenbrink. 2006. Spatial reference in linguistic human-robot interaction: Iterative, empirically supported development of a model of projective relations. Spatial Cognition and Computation.

L. Talmy. 1983. How language structures space. In H.L. Pick, editor, Spatial orientation. Theory, research and application, pages 225-282. Plenum Press.

A. Treisman and S. Gormican. 1988. Feature analysis in early vision: Evidence from search assymetries. Psychological Review, 95:15-48.

K. van Deemter. 2001. Generating referring expressions: Beyond the incremental algorithm. In 4th Int. Conf. on Computational Semantics (IWCS-4), Tilburg.

I van der Sluis and E Krahmer. 2004. The influence of target size and distance on the production of speech and gesture in multimodal referring expressions. In Proceedings of International Conference on Spoken Language Processing (ICSLP04).

C. Vandeloise. 1991. Spatial Prepositions: A Case Study From French. The University of Chicago Press.

S. Varges. 2004. Overgenerating referring expressions involving relations and booleans. In Proceedings of the $3 \mathrm{rd}$ International Conference on Natural Language Generation, University of Brighton. 